\title{
Detecting the first quasars with ALMA
}

\section{Dominik R. G. Schleicher ${ }^{1,2,3}$, Marco Spaans ${ }^{4}$ and Ralf S. Klessen ${ }^{1}$}

${ }^{1}$ Zentrum für Astronomie der Universität Heidelberg, Institut für Theoretische Astrophysik, Albert-Ueberle-Str. 2, 69120 Heidelberg, Germany. E-Mail: dschleic@ita.uni-heidelberg.de

${ }^{2}$ Leiden Observatory, P.O. Box 9513, NL-2300 RA Leiden, the Netherlands

${ }^{2}$ ESO Garching, Karl-Schwarzschild-Str. 2, 85748 Garching bei München, Germany

${ }^{4}$ Kapteyn Astronomical Institute, University of Groningen, P.O. Box 800, 9700 AV, Groningen, the Netherlands

\begin{abstract}
We show that ALMA is the first telescope that can probe the (dust) obscured central region of quasars at $z>5$ with a maximum resolution of $\sim 30 \mathrm{pc}$ employing the $18 \mathrm{~km}$ baseline.
\end{abstract}

Keywords. astrochemistry - telescopes - galaxies: active - high-redshift - ISM

We explore the possibility to detect the first quasars with ALMA - Schleicher, Spaans \& Klessen(2009). For this purpose, we adopt the Seyfert 2 galaxy NGC 1068 as a reference system and calculate the expected fluxes if this galaxy were placed at high redshift. This choice seems justified due to the absence of any indication for an evolution in metallicity in high-redshift quasars, and is conservative due to the moderate column densities in NGC 1068, leading to correspondingly moderate fluxes.

On large scales, the dominant excitation mechamism are soft UV-photons produced by the starburst, leading to strong emission in the [CII] $158 \mu \mathrm{m}$ and the [OI] $63 \mu \mathrm{m}$ line. Due to its unprecedented angular resolution, ALMA can probe the central $200 \mathrm{pc}$ of high-redshift quasars. Calculations with the X-ray dominated region code of Meijerink \& Spaans(2005) show that strong emission in the high- $J$ CO lines can be expected in these regions (see Fig. 1). As these lines are redshifted into the ALMA bands, this may provide the first opportunity to detect these lines. Flux estimates for various fine-structure lines are provided in the paper.

\section{References}

Meijerink, R. \& Spaans, M., 2005, A\&A, 436, 397

Schleicher, D. R. G., Spaans, M., \& Klessen, R. S., 2009, submitted to A\&A

$\dagger$ Member of the Carrera del Investigador Cient ‘fico, CONICET, Argentina.

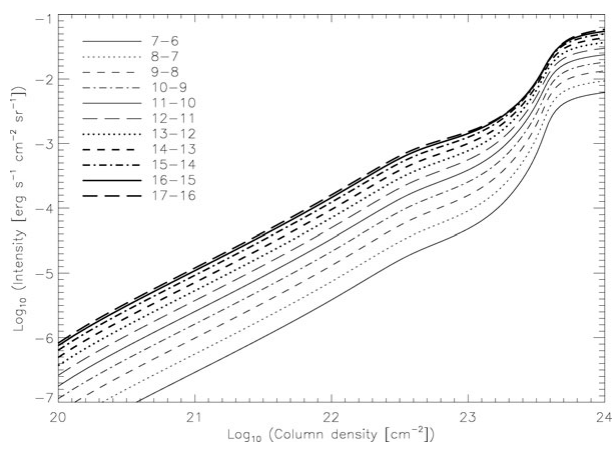

Figure 1. The expected intensities in the high- $J$ CO lines as a function of the cloud column density in the central region of a system like NGC 1068. 